\title{
Prognostic Value of Hematological Parameters in Locally Advanced Cervical Cancer Patients Treated With Concurrent Chemoradiotherapy
}

\author{
HYUN JUNG LEE ${ }^{1,2}$, JONG MI KIM ${ }^{1,2}$, YOO JEONG CHIN ${ }^{1,2}$, GUN OH CHONG ${ }^{1,3,4}$, \\ SHIN-HYUNG PARK ${ }^{5,6}$, YOON HEE LEE ${ }^{1,3,4}$, DAE GY HONG ${ }^{1,3}$ and YOON SOON LEE ${ }^{1,3}$ \\ ${ }^{1}$ Department of Obstetrics and Gynecology, School of Medicine, \\ Kyungpook National University, Daegu, Republic of Korea; \\ ${ }^{2}$ Department of Obstetrics and Gynecology, Kyungpook National University Hospital, Daegu, Republic of Korea; \\ ${ }^{3}$ Department of Obstetrics and Gynecology, \\ Kyungpook National University Chilgok Hospital, Daegu, Republic of Korea, \\ ${ }^{4}$ Molecular Diagnostics and Imaging Center, School of Medicine, \\ Kyungpook National University, Daegu, Republic of Korea; \\ ${ }^{5}$ Department of Radiation Oncology, School of Medicine, \\ Kyungpook National University, Daegu, Republic of Korea; \\ ${ }^{6}$ Department of Radiation Oncology, Kyungpook National University Hospital, Daegu, Republic of Korea
}

\begin{abstract}
Background/Aim: We evaluated the clinical implications of pre- and post-treatment hematological parameters as prognostic factors in patients with locally advanced cervical cancer (LACC) who received definitive concurrent chemoradiotherapy (CCRT). Patients and Methods: We retrospectively analyzed 125 patients with LACC (FIGO stage IIB to IIIB) who received definitive CCRT. Clinical factors and hematological parameters, including neutrophil-to-lymphocyte ratio (NLR) were assessed pre- and post-CCRT. Univariate and multivariate analysis for diseasefree survival (DFS) and overall survival (OS) were performed using clinicopathological and hematological parameters. Results: Disease recurred in 46 (36.8\%) patients, and 24 patients (19.2\%) died. On multivariate analysis, posttreatment NLR, $\triangle N L R$ (pre-treatment NLR/post-treatment $N L R$ ) and $\triangle P L R$ (platelet-to-lymphocyte ratio) (pretreatment $P L R /$ post-treatment PLR) were significant prognostic factors
\end{abstract}

Correspondence to: Gun Oh Chong, MD, Ph.D., Associate Professor, Department of Obstetrics and Gynecology, School of Medicine, Kyungpook National University, Kyungpook National University Chilgok Hospital, 807, Hoguk-ro, Buk-gu, Daegu, 41404, Republic of Korea. Tel: +82 532002884, Fax: +82 532002028, e-mail: gochong@knu.ac.kr

Key Words: Locally advanced cervical cancer, post-treatment hematological parameters, prognosis, neutrophil-to-lymphocyte, concurrent chemoradiotherapy. for DFS, and only post-treatment NLR was a significant prognostic factor for $O S(p<0.001)$. However, pre-treatment hematological parameters were not associated with prognosis. Conclusion: Post-treatment hematological parameters, particularly NLR, may serve as a prognostic indicator in patients with $L A C C$ who received definitive CCRT.

Cervical cancer is the fourth most frequently diagnosed cancer and the fourth leading cause of cancer-related deaths in women, accounting for 570,000 new cases and over 300,000 deaths worldwide (1). For patients with locally advanced cervical cancer (LACC), definitive concurrent chemoradiotherapy (CCRT) using a cisplatin-based regimen has become the standard treatment. However, about one third of patients with cervical cancer experience recurrence, and recurrence mostly develops within 2 years of therapy completion (2). Therefore, accurate prognosis prediction is important to improve the survival of patients who may benefit from adjuvant treatment or close surveillance. The relationship of inflammation with cancer development and progression is widely accepted, and diverse prognostic markers based on systemic inflammation have been evaluated to predict prognosis in cancer patients (3). In particular, high peripheral neutrophil-to-lymphocyte ratio (NLR) has been recognized as a poor prognostic indicator in various cancers, including cervical cancer (4-12). Recently, post-treatment inflammatory hematological markers and their changes between pre-treatment and post-treatment time points have been reported to be associated with prognosis in 
Table I. Clinicopathological characteristics of patients.

\begin{tabular}{lccc}
\hline & $\begin{array}{c}\text { Total } \\
(\mathrm{n}=125)\end{array}$ & $\begin{array}{c}\text { No recurrence } \\
(\mathrm{n}=80)\end{array}$ & $\begin{array}{c}\text { Recurrence } \\
(\mathrm{n}=45)\end{array}$ \\
\hline Age (years) & $53.67 \pm 13.23$ & $55.21 \pm 13.62$ & $50.93 \pm 12.18$ \\
Histology & & & $39(31.2)$ \\
Squamous cell carcinoma & $114(91.2)$ & $75(60.0)$ & $5(4.0)$ \\
$\quad$ Adenocarcinoma & $10(8.0)$ & $5(4.0)$ & $1(0.8)$ \\
Adenosquamous cell carcinoma & $1(0.8)$ & $0(0)$ & $32(25.6)$ \\
FIGO stage (n, \%) & $106(84.8)$ & $74(59.2)$ & 0.074 \\
IIB & $3(2.4)$ & $3(2.4)$ & $4(3.2)$ \\
IIIA & $6(4.8)$ & $2(1.6)$ & $9(7.2)$ \\
IIIB & $10(8.0)$ & $1(0.8)$ & $5.02 \pm 1.73$ \\
IIIC & $4.49 \pm 1.57$ & $4.19 \pm 1.40$ & $33(26.4)$ \\
Primary tumor size (cm) & $78(62.4)$ & $45(36.0)$ & $10(8.0)$ \\
Lymph node metastasis $(\mathrm{n}, \%)$ & $17(13.6)$ & $7(5.6)$ & $21.14 \pm 26.41$ \\
Pelvis & $18.87 \pm 34.53$ & $17.53 \pm 38.62$ & 0.037 \\
Paraaortic & & & 0.004 \\
SCC antigen $(\mathrm{mg} / \mathrm{ml})$ & & & 0.053 \\
\hline
\end{tabular}

FIGO: International Federation of Gynecology and Obstetrics; SCC: squamous cell carcinoma.

various cancers (13-17). However, the consistency of the prognostic impact of NLR in cervical cancer has not been determined, and most studies emphasize the importance of pre-treatment NLR $(7,18-20)$. To our knowledge, there are no studies on the prognostic impact of post-treatment hematological parameters and post-treatment changes in cervical cancer. The aim of this study was to assess the prognostic value of pre-treatment and post-treatment hematological parameters and their changes after definitive CCRT in patients with LACC.

\section{Patients and Methods}

Patients. We retrospectively analyzed 125 patients with cervical cancer classified as having stage IIB to IIIB cancer; these patients were treated with definitive CCRT with a cisplatin-based regimen between September 2005 and November 2016 at Kyungpook National University Hospital. Retrospective data collection and analysis were approved by the institutional review board of Kyungpook National University Chilgok Hospital. The need for informed consent was waived due to the retrospective design of the study. Clinical and pathological parameters were reviewed and retrieved, including age, International Federation of Gynecology and Obstetrics (FIGO) stage, histology, primary tumor size, pelvic and paraaortic lymph node metastasis, and squamous cell carcinoma (SCC) antigen.

The patients were staged according to the 2008 FIGO staging system (21). Tumor size was determined by measuring the largest tumor diameter in MRI or CT images. Lymph node metastasis was defined as having any pelvic or para-aortic LN with a diameter greater than $1 \mathrm{~cm}$ along the short axis on MRI or CT.

Treatment. All patients were treated with a combination of external beam radiotherapy (EBRT) and high-dose-rate (HDR) intracavitary brachytherapy (ICR) with curative intent. EBRT was delivered to the whole pelvis using a 3-dimensional conformal radiation therapy (3D-CRT) 4-field box technique (1.8 Gy daily fractions, five times a week; total dose, 45 Gy). Extended-field radiotherapy, including the pelvis and paraaortic nodal area, was delivered to patients with paraaortic nodal involvement. A parametrial boost of $10 \mathrm{~Gy}$ in five fractions was additionally given to patients with parametrial involvement. HDR ICR was initiated after the delivery of an EBRT dose of $39.6 \mathrm{~Gy}$. ICR was delivered twice a week in five fractions with a fractional dose of 6 Gy at point A. Cisplatin-based CCRT regimens included weekly cisplatin $\left(40 \mathrm{mg} / \mathrm{m}^{2}\right)$, OMP (vincristine $1 \mathrm{mg} / \mathrm{m}^{2}+$ mitomycin $10 \mathrm{mg} / \mathrm{m}^{2}+$ cisplatin $75 \mathrm{mg} / \mathrm{m}^{2}$ ) or $\mathrm{CP}$ (cyclophosphamide $500 \mathrm{mg} / \mathrm{m}^{2}+$ cisplatin $50 \mathrm{mg} / \mathrm{m}^{2}$ ). The first course of cisplatin was administered on day 1 of radiotherapy.

Evaluation of the hematologic parameters. A routine blood examination was performed before starting CCRT and after CCRT (between 2 and 4 weeks after completion of chemotherapy). Hematological parameters included absolute white blood cell (WBC) counts (AWC), absolute lymphocyte counts (ALC), absolute neutrophil counts (ANC), absolute monocyte counts (AMC), and absolute platelet counts (ACP). Neutrophil-to-lymphocyte ratio (NLR) was calculated by dividing ANC by ALC. Monocyte-tolymphocyte ratio (MLR) and platelet-to-lymphocyte ratio (PLR) were similarly calculated. $\triangle \mathrm{NLR}$ was defined as follows: $\triangle \mathrm{NLR}=$ pre-treatment NLR/post-treatment NLR. $\triangle \mathrm{MLR}$ and $\triangle \mathrm{PLR}$ were defined similarly.

Clinical follow-up. Patients were followed up with physical examinations and Pap smears every 3 months for 2 years and then every 6 months for the next 3 years. Chest radiography and imaging studies, such as pelvic CT or positron emission tomography/CT, were performed annually. Complete remission was defined as no evidence of disease at 6 months after diagnosis. Failure was defined as biopsy-proven recurrence or documentation of disease progression on serial imaging studies. Disease-free survival (DFS) was defined as the time from initial treatment to relapse noted on images or in histologic examinations or as the time to the last 
Lee et al: Prognostic Value of Post-treatment Hematological Parameters in Locally Advanced Cervical Cancer Patients

Table II. Pre- and post-treatment hematological parameters of patients with or without recurrence.

\begin{tabular}{|c|c|c|c|}
\hline \multirow[t]{2}{*}{ Variable } & \multirow{2}{*}{$\frac{\text { No recurrence }}{\text { Mean } \pm \text { SD }}$} & \multirow{2}{*}{$\begin{array}{l}\text { Recurrence } \\
\text { Mean } \pm \text { SD }\end{array}$} & \multirow[t]{2}{*}{$p$-Value } \\
\hline & & & \\
\hline \multicolumn{4}{|c|}{ Pre-CCRT hematological parameters } \\
\hline WBC $\left(10^{3} / \mu \mathrm{l}\right)$ & $8.049 \pm 3.252$ & $8.192 \pm 3.591$ & 0.820 \\
\hline Lymphocyte $\left(10^{3} / \mu \mathrm{l}\right)$ & $1.812 \pm 0.559$ & $1.872 \pm 0.757$ & 0.610 \\
\hline Neutrophil $\left(10^{3} / \mu \mathrm{l}\right)$ & $5.547 \pm 3.009$ & $5.657 \pm 3.163$ & 0.848 \\
\hline Monocyte $\left(10^{3} / \mu \mathrm{l}\right)$ & $0.373 \pm 0.144$ & $0.411 \pm 0.202$ & 0.219 \\
\hline Platelet $\left(10^{3} / \mu \mathrm{l}\right)$ & $309.563 \pm 90.748$ & $325.933 \pm 130.437$ & 0.458 \\
\hline NLR & $3.782 \pm 4.989$ & $3.336 \pm 1.983$ & 0.567 \\
\hline MLR & $0.238 \pm 0.201$ & $0.243 \pm 0.129$ & 0.869 \\
\hline PLR & $193.632 \pm 114.668$ & $192.811 \pm 88.747$ & 0.967 \\
\hline \multicolumn{4}{|c|}{ Post-CCRT hematological parameters } \\
\hline $\operatorname{WBC}\left(10^{3} / \mu \mathrm{l}\right)$ & $3.070 \pm 1.224$ & $3.303 \pm 1.338$ & 0.325 \\
\hline Lymphocyte $\left(10^{3} / \mu \mathrm{l}\right)$ & $0.690 \pm 0.342$ & $0.451 \pm 0.194$ & 0.001 \\
\hline Neutrophil $\left(10^{3} / \mu \mathrm{l}\right)$ & $1.857 \pm 0.800$ & $2.465 \pm 1.179$ & $<0.001$ \\
\hline Monocyte $\left(10^{3} / \mu \mathrm{l}\right)$ & $0.272 \pm 0.120$ & $0.251 \pm 0.099$ & 0.321 \\
\hline Platelet $\left(10^{3} / \mu \mathrm{l}\right)$ & $208.825 \pm 65.616$ & $196.067 \pm 71.475$ & 0.314 \\
\hline NLR & $3.056 \pm 1.571$ & $6.121 \pm 3.095$ & $<0.001$ \\
\hline MLR & $0.453 \pm 0.247$ & $0.636 \pm 0.337$ & 0.002 \\
\hline PLR & $365.766 \pm 205.864$ & $497.817 \pm 255.480$ & 0.002 \\
\hline \multicolumn{4}{|c|}{ Change of hematological parameters } \\
\hline$\triangle \mathrm{NLR}$ & $2.312 \pm 1.396$ & $3.048 \pm 1.654$ & 0.009 \\
\hline$\triangle \mathrm{MLR}$ & $1.185 \pm 0.900$ & $2.243 \pm 1.344$ & $<0.001$ \\
\hline$\triangle \mathrm{PLR}$ & $2.138 \pm 1.168$ & $3.281 \pm 3.849$ & 0.058 \\
\hline
\end{tabular}

HR: Hazard ratio; CI: confidence interval; CCRT: concurrent chemoradiotherapy; NLR: neutrophil-to-lymphocyte ratio; MLR: monocyte-tolymphocyte ratio; PLR: platelet-to-lymphocyte ratio; $\triangle \mathrm{NLR}$ : pre-treatment NLR/post-treatment NLR; $\triangle \mathrm{MLR}$ : pre-treatment MLR/post-treatment MLR; $\triangle$ PLR: pre-treatment PLR/post-treatment PLR.

follow-up visit. Overall survival (OS) was defined as the period from initial treatment to the last follow-up visit or the date of death.

Statistical analysis. The time to event was calculated as the time interval from the date of diagnosis to the date of the first finding suggesting disease recurrence on clinical or imaging examination. The differences between subsets were evaluated with the Student's $t$-test, and differences between proportions were compared with the chi-square test. To identify an optimal cutoff of hematologic parameters for the prediction of recurrence and death, receiver operating characteristics (ROC) curve analysis was performed. The Kaplan-Meier method and the log-rank test were used in the survival analysis of prognostic factors. The Cox proportional hazard model was used to evaluate prognostic variables for univariate and multivariate comparisons, and herein, an estimated hazard ratio (HR) with $95 \%$ confidence intervals (CI) is presented. Statistical analyses were performed using SPSS 21.0 for Windows (IBM Corporation, Armonk, NY, USA), and $p$-values $<0.05$ were considered to indicate statistically significant differences.

\section{Results}

Patient characteristics. The clinical characteristics of the 125 cervical cancer patients with stage IIB to IIIB disease who received primary CCRT are summarized in Table I. One hundred fourteen patients with squamous cell carcinoma
(91.2\%), 10 patients $(8.0 \%)$ with adenocarcinoma, and one patient $(0.8 \%)$ with adenosquamous cell carcinoma were included in this study. The mean age was $53.67 \pm 13.23$ years.

The mean tumor size was $4.49 \pm 1.57 \mathrm{~cm}$. The mean SCC antigen level was $18.87 \pm 34.53 \mathrm{mg} / \mathrm{ml}$. FIGO stages were as follows: $106(84.8 \%)$ patients, IIB; three patients $(2.4 \%)$, IIIA1; six patients $(4.8 \%)$, IIIA2; and 10 patients $(8.0 \%)$, IIIB. Seventy-eight (62.4\%) patients had pelvic lymph node metastasis, and 17 (13.6\%) patients had paraaortic lymph node metastasis. A total of $45(36.0 \%)$ patients showed disease recurrence, and 24 (19.2) patients died after a median follow-up duration of 50 months (range=6-124 months) (Table I).

Pre-treatment and post-treatment hematological parameters. There were no significant differences in the pretreatment hematological parameters between the no-recurrence group and the recurrence group; however, post-treatment hematological parameters, including ALC $(p=0.001)$, ANC $(p<0.001)$, NLR $(p<0.001), \quad \operatorname{MLR}(p=0.002), \quad$ PLR $\quad(p=0.002), \quad \Delta \mathrm{NLR}$ $(p=0.009)$, and $\triangle$ MLR $(p<0.001)$, showed differences between the disease-free group and the recurrence group (Table II). Post-treatment hematological parameters, including AWC $(p=0.043)$, ALC $(p<0.001)$, ANC $(p=0.006)$, NLR $(p<0.001)$, 
A

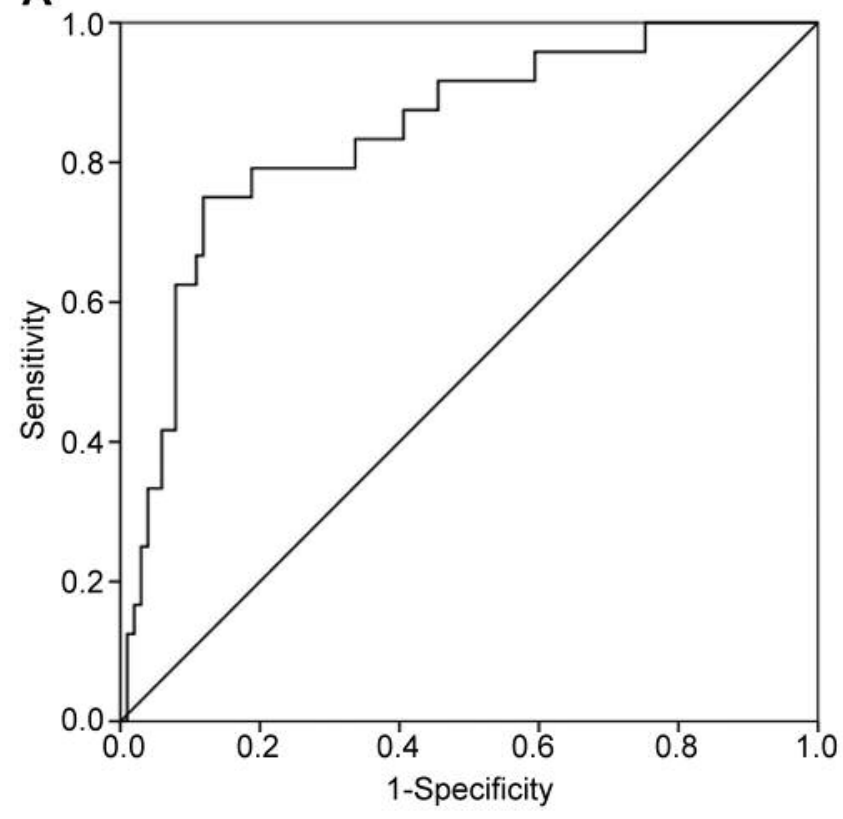

B

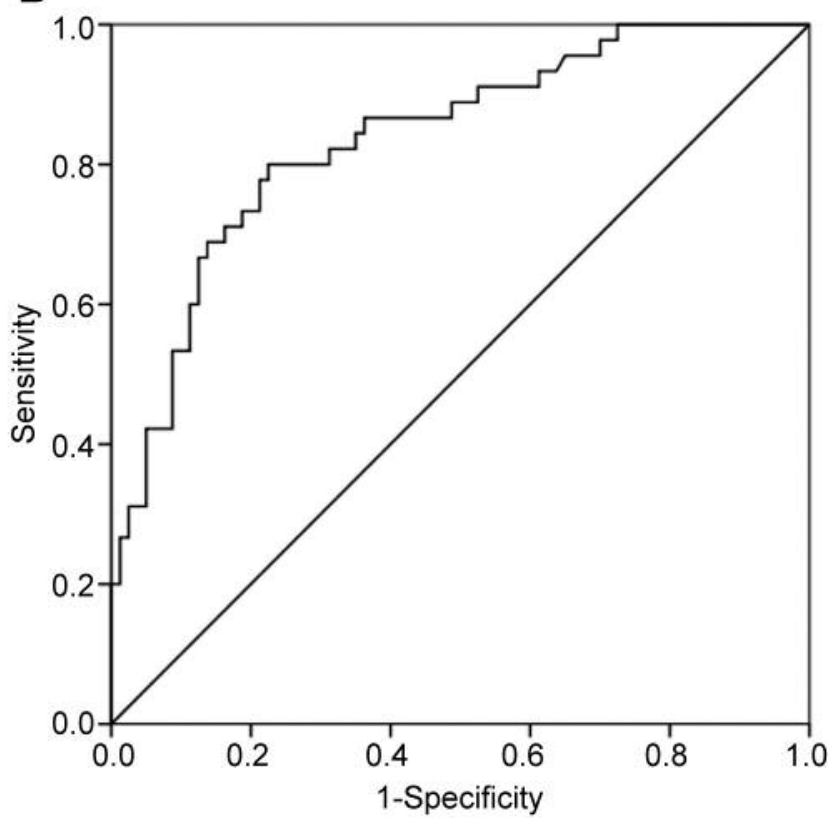

Figure 1. Evaluation of post-treatment NLR as prognostic indicator using receiver operating characteristic (ROC) curves. A. Disease-free survival, $B$. Overall survival.

$\operatorname{MLR}(p=0.023), \operatorname{PLR}(p=0.021)$, and $\Delta \operatorname{NLR}(p=0.001)$ showed significant differences between disease-free and recurrence group survival or death group.

Cut-off value of metabolic parameters. The optimal cut-off values for each hematological parameter were calculated using the ROC curve. The ROC curves suggested that posttreatment NLR was the best prognostic indicator of OS and DFS. The areas under the curves (AUCs) of post-treatment NLR were 0.830 for DFS and 0.842 for OS (Figure 1).

Univariate and multivariate analyses for DFS and OS. Univariate analysis revealed that tumor size, stage, paraaortic lymph node metastasis, SCC level, pretreatment AMC and PLR, post-treatment ALC, ANC, NLR, PLR and MLR, $\triangle P L R, \triangle N L R$, and $\triangle$ MLR were significant predictors of DFS. Multivariate analysis confirmed that elevated posttreatment NLR $(\mathrm{HR}=3.643,95 \% \mathrm{CI}=1.701-7.802 ; p<0.001)$, $\triangle \mathrm{NLR}(\mathrm{HR}=3.024,95 \% \mathrm{CI}=1.343-6.809 ; p=0.008), \Delta \mathrm{PLR}$ $(\mathrm{HR}=2.077,95 \% \mathrm{CI}=1.044-4.133 ; p=0.037)$, paraaortic lymph node metastasis $(\mathrm{HR}=2.904,95 \% \mathrm{CI}=1.349-6.251 ; p=0.006)$, and tumor size $(\mathrm{HR}=6.819,95 \% \mathrm{CI}=2.833-16.413 ; p<0.001)$ were independent predictors of DFS (Table III). For OS, age, tumor size, paraaortic lymph node metastasis, pretreatment AWC, ALC, ANC, AMC, PLR, MLR and post-treatment AWC, ALC, ANC, NLR, PLR, MLR, $\triangle \mathrm{PLR}$, and $\triangle \mathrm{NLR}$ were significant predictors in univariate analysis. In multivariate analysis, only elevated post-treatment NLR
$(\mathrm{HR}=12.639,95 \% \mathrm{CI}=4.969-32.144 ; p<0.001)$ and tumor size $(\mathrm{HR}=5.025,95 \% \mathrm{CI}=1.4846-17.008 ; p=0.010)$ were useful markers in predicting OS (Table IV).

Survival analyses. The patients were divided into two subgroups according to cut-off values of the post-treatment NLRs. The differences in DFS and OS outcomes between the two subgroups are presented in Figure 2.

\section{Discussion}

In this study, we evaluated the prognostic value of pre- and post-treatment hematological parameters in LACC patients who were treated with definitive CCRT. Pre-treatment hematological parameters were not associated with prognosis; however, post-treatment hematological parameters, particularly high NLR, were independent prognostic factors, which predicted recurrence and death.

Increasing evidence suggests that there is an association between cancer progression and systemic inflammation (22). Among variable inflammatory markers, NLR has been frequently used as a prognostic factor in various tumors during the last decade. The impact of high pre-treatment NLR on poor prognosis in various cancers, including cervical cancer, has been reported in a recent meta-analysis $(18,23,24)$; however, the significance of post-treatment NLR after removing the tumor was still uncertain. Moreover, to our knowledge, no study has reported on the prognostic 
Table III. Univariate and multivariate analysis of factors affecting disease-free survival.

\begin{tabular}{|c|c|c|c|c|c|c|c|c|c|}
\hline \multirow[b]{2}{*}{ Variable } & \multirow[b]{2}{*}{ Cut-off } & \multirow[b]{2}{*}{ AUC } & \multirow[b]{2}{*}{$p$-Value } & \multicolumn{3}{|c|}{ Univariate } & \multicolumn{3}{|c|}{ Multivariate } \\
\hline & & & & HR & $95 \% \mathrm{CI}$ & $p$-Value & HR & $95 \% \mathrm{CI}$ & $p$-Value \\
\hline Age (years) & $\leq 66$ & 0.565 & 0.216 & 1.937 & $0.914-4.106$ & 0.085 & & & \\
\hline Pathology & $\mathrm{AC}$ & & & 1.783 & $0.630-5.048$ & 0.276 & & & \\
\hline Tumor size & $\geq 4$ & 0.641 & 0.006 & 2.844 & $1.565-5.167$ & 0.001 & 6.819 & $2.833-16.413$ & $<0.001$ \\
\hline Stage & $>\mathrm{IIB}$ & & & 4.600 & $1.887-11.213$ & 0.001 & & & \\
\hline Pelvic LN metastasis & & & & 1.674 & $0.924-3.033$ & 0.089 & & & \\
\hline Paraaortic LN metastasis & & & & 3.121 & $1.222-7.973$ & 0.017 & 2.904 & $1.349-6.251$ & 0.006 \\
\hline SCC antigen level & $>10.8$ & 0.601 & 0.072 & 2.748 & $1.448-5.216$ & 0.002 & & & \\
\hline \multicolumn{10}{|c|}{ Pre-CCRT hematologic markers } \\
\hline WBC & $>7.75$ & 0.509 & 0.872 & 1.117 & $0.623-2.004$ & 0.711 & & & \\
\hline Lymphocyte & $>5$ & 0.516 & 0.773 & 1.426 & $0.797-2.561$ & 0.231 & & & \\
\hline Neutrophil & $\leq 1.76$ & 0.500 & 0.994 & 5.027 & $0.696-2.236$ & 0.458 & & & \\
\hline Monocyte & $>0.43$ & 0.547 & 0.402 & 2.076 & $1.063-4.052$ & 0.032 & & & \\
\hline Platelet & $\leq 441$ & 0.500 & 0.996 & 2.548 & $0.669-9.705$ & 0.171 & & & \\
\hline NLR & $>3.04$ & 0.501 & 0.992 & 1.264 & $0.697-2.290$ & 0.440 & & & \\
\hline MLR & $>174.30$ & 0.533 & 0.542 & 1.489 & $0.830-2.671$ & 0.182 & & & \\
\hline PLR & $\leq 3.85$ & 0.546 & 0.413 & 2.582 & $1.324-5.037$ & 0.005 & & & \\
\hline \multicolumn{10}{|c|}{ Post-CCRT hematologic markers } \\
\hline WBC & $>2.6$ & 0.549 & 0.353 & 2.017 & $0.802-5.076$ & 0.136 & & & \\
\hline Lymphocyte & $>1.89$ & 0.664 & 0.001 & 2.521 & $1.405-4.524$ & 0.002 & & & \\
\hline Neutrophil & $\leq 0.52$ & 0.748 & $<0.001$ & 3.471 & $1.928-6.249$ & $<0.001$ & & & \\
\hline Monocyte & $>0.48$ & 0.559 & 0.256 & 1.580 & $0.346-7.214$ & 0.555 & & & \\
\hline Platelet & $\leq 159$ & 0.540 & 0.467 & 1.702 & $0.865-3.351$ & 0.124 & & & \\
\hline NLR & $>3.76$ & 0.83 & $<0.001$ & 7.339 & $3.960-13.602$ & $<0.001$ & 3.643 & $1.701-7.802$ & 0.001 \\
\hline MLR & $>356.60$ & 0.682 & $<0.001$ & 3.145 & $1.754-5.637$ & $<0.001$ & & & \\
\hline PLR & $\leq 2.31$ & 0.663 & 0.001 & 3.136 & $1.745-5.634$ & $<0.001$ & & & \\
\hline \multicolumn{10}{|c|}{$\begin{array}{l}\text { Change of hematologic markers } \\
\text { between pre-CCRT and post-CCRT }\end{array}$} \\
\hline$\triangle N L R$ & $\leq 0.81$ & 0.781 & $<0.001$ & 4.613 & $2.554-8.332$ & $<0.001$ & 3.024 & $1.343-6.809$ & 0.008 \\
\hline$\triangle \mathrm{MLR}$ & $\leq 0.38$ & 0.638 & 0.007 & 1.857 & $1.018-3.387$ & 0.043 & & & \\
\hline$\triangle \mathrm{PLR}$ & $>2.57$ & 0.656 & 0.003 & 3.188 & $1.727-5.886$ & $<0.001$ & 2.077 & $1.044-4.133$ & 0.037 \\
\hline
\end{tabular}

CCRT: Concurrent chemoradiotherapy; NLR: neutrophil-to-lymphocyte ratio; MLR: monocyte-to-lymphocyte ratio; PLR: platelet-to-lymphocyte ratio; $\triangle$ NLR: pre-treatment NLR/post-treatment NLR; $\triangle$ MLR: pre-treatment MLR/post-treatment MLR; $\triangle \mathrm{PLR}$ : pre-treatment PLR/post-treatment PLR.

value of post-treatment hematological parameters in cervical cancer. Therefore, in this study, we focused on determining the predictive significance of post-treatment hematological parameters in patients with LACC.

As hypothesized by Rudolf Virchow, there is a significant relationship between inflammation and cancer (25). As the relationship between NLR and survival is complicated, the specific mechanism involved in the interaction between high NLR and poor prognosis has not been completely defined. Neutrophils are the dominant inflammatory cells in the blood and are considered to be the primary source of vascular endothelial growth factor, which plays a critical role in tumor-related angiogenesis by inducing the release of cytokines, chemokines, and granule proteins. This way, neutrophils provide a favorable microenvironment for tumor growth and progression $(3,22,26)$. Conversely, lymphocytes play an important role in the antitumor immunological reaction, which may be associated with protection against the development and progression of cancer $(27,28)$. High NLR is caused by an increase in neutrophil count and/or a decrease in lymphocyte count and indicates the development of a favorable immune microenvironment for tumor progression and metastasis which leads to a worse prognosis.

Although it is known that high pretreatment NLR is associated with a poor outcome in various cancer types (6$11,18,23)$, the relationship between post-treatment NLR and prognosis has not been well defined. Recently, several studies have reported that high post-treatment NLR is associated with poor outcomes in patients treated by radiotherapy due to locally advanced cancer (13-17). In a study of oropharyngeal cancer wherein the patients had been treated by radiotherapy, the ALC and NLR at 3 months after the initiation of radiotherapy were found to be associated with DFS and OS (14). Furthermore, in another 
Table IV. Univariate and multivariate analysis of factors affecting overall survival.

\begin{tabular}{|c|c|c|c|c|c|c|c|c|c|}
\hline \multirow[b]{2}{*}{ Variable } & \multirow[b]{2}{*}{ Cut-off } & \multirow[b]{2}{*}{ AUC } & \multirow[b]{2}{*}{$p$-Value } & \multicolumn{3}{|c|}{ Univariate } & \multicolumn{3}{|c|}{ Multivariate } \\
\hline & & & & HR & $95 \% \mathrm{CI}$ & $p$-Value & HR & $95 \% \mathrm{CI}$ & $p$-Value \\
\hline Age (years) & $\leq 49$ & 0.639 & 0.031 & 2.566 & $1.115-5.904$ & 0.027 & & & \\
\hline Pathology & $\mathrm{AC}$ & & & 1.383 & $0.356-5.369$ & 0.639 & & & \\
\hline Tumor size & $>4.4$ & 0.636 & 0.036 & 2.973 & $1.331-6.641$ & 0.008 & 5.025 & $1.485-17.008$ & 0.010 \\
\hline Stage & $>$ IIB & & & 3.086 & $0.923-10.323$ & 0.067 & & & \\
\hline Pelvic LN metastasis & & & & 1.039 & $0.456-2.371$ & 0.927 & & & \\
\hline Paraaortic LN metastasis & & & & 3.467 & $1.006-11.952$ & 0.049 & & & \\
\hline SCC antigen level & $>28$ & 0.6 & 0.148 & 2.554 & $0.918-7.106$ & 0.072 & & & \\
\hline \multicolumn{10}{|c|}{ Pre-CCRT hematologic markers } \\
\hline WBC & $>11.34$ & 0.553 & 0.454 & 8.597 & $1.778-41.570$ & 0.008 & & & \\
\hline Lymphocyte & $>7.21$ & 0.543 & 0.552 & 3.303 & $1.129-9.669$ & 0.029 & & & \\
\hline Neutrophil & $\leq 1.61$ & 0.529 & 0.676 & 2.368 & $1.018-5.511$ & 0.008 & & & \\
\hline Monocyte & $>0.33$ & 0.629 & 0.041 & 2.970 & $1.322-6.673$ & 0.004 & & & \\
\hline Platelet & $>389$ & 0.545 & 0.526 & 2.073 & $0.812-5.290$ & 0.127 & & & \\
\hline NLR & $>2.659$ & 0.539 & 0.547 & 1.909 & $0.853-4.273$ & 0.116 & & & \\
\hline MLR & $>174.270$ & 0.557 & 0.386 & 3.784 & $1.613-8.874$ & 0.002 & & & \\
\hline PLR & $\leq 4.237$ & 0.588 & 0.174 & 3.143 & $1.309-7.544$ & 0.010 & & & \\
\hline \multicolumn{10}{|c|}{ Post-CCRT hematologic markers } \\
\hline WBC & $>3.38$ & 0.634 & 0.041 & 3.217 & $1.369-7.561$ & 0.007 & & & \\
\hline Lymphocyte & $>2.76$ & 0.73 & $<0.001$ & 12.206 & $4.109-36.252$ & $<0.001$ & & & \\
\hline Neutrophil & $\leq 0.62$ & 0.714 & $<0.001$ & 3.840 & $1.695-8.699$ & 0.001 & & & \\
\hline Monocyte & $\leq 0.31$ & 0.572 & 0.266 & 1.180 & $0.484-2.874$ & 0.716 & & & \\
\hline Platelet & $\leq 259$ & 0.53 & 0.652 & 2.448 & $0.964-6.215$ & 0.060 & & & \\
\hline NLR & $>5.231$ & 0.842 & $<0.001$ & 28.720 & $10.631-77.589$ & $<0.001$ & 12.639 & $4.969-32.144$ & $<0.001$ \\
\hline MLR & $>368$ & 0.663 & 0.008 & 3.402 & $1.522-7.606$ & 0.003 & & & \\
\hline PLR & $\leq 2.235$ & 0.687 & 0.001 & 4.257 & $1.907-9.504$ & $<0.001$ & & & \\
\hline \multicolumn{10}{|c|}{$\begin{array}{l}\text { Change of hematologic markers } \\
\text { between pre-CCRT and post-CCRT }\end{array}$} \\
\hline$\Delta \mathrm{NLR}$ & $\leq 0.800$ & 0.763 & $<0.001$ & 5.519 & $2.463-12.365$ & $<0.001$ & & & \\
\hline$\triangle \mathrm{MLR}$ & $\leq 0.580$ & 0.579 & 0.195 & 1.627 & $0.706-3.749$ & 0.254 & & & \\
\hline$\triangle \mathrm{PLR}$ & $>1.824$ & 0.618 & 0.047 & 3.016 & $1.280-7.107$ & 0.012 & & & \\
\hline
\end{tabular}

CCRT: Concurrent chemoradiotherapy; NLR: neutrophil-to-lymphocyte ratio; MLR: monocyte-to-lymphocyte ratio; PLR: platelet-to-lymphocyte ratio; $\triangle$ NLR: pre-treatment NLR/post-treatment NLR; $\triangle$ MLR: pre-treatment MLR/post-treatment MLR; $\triangle P L R$ : pre-treatment PLR/post-treatment PLR.

study of patients with head and neck cancer, post-treatment NLR was reported to be superior to pretreatment NLR in predicting recurrence and overall survival (17). In esophageal (16) and locally advanced lung cancer (15), high post-treatment NLR values have been associated with decreased DFS and OS. Pretreatment NLR reflects the baseline balance between pro-tumor and anti-tumor, while post-treatment NLR can represent the status after tumor removal, which would be expected to provide a more precise indication of treatment efficacy and prediction of recurrence and survival (17).

In contrast to previous studies $(7,8,10-12)$, no significant prognostic value of pre-treatment NLR was observed in our study. In this study, however, we found that post-treatment hematological parameters, particularly high post-treatment NLR, were associated with poor DFS and OS in patients with LACC who received definitive CCRT. To our knowledge, this is the first study to evaluate the prognostic significance of post-treatment NLR in patients with LACC who have undergone definitive CCRT. Peripheral blood cell counting is carried out routinely at a low cost during the treatment and follow-up of cancer patients; thus, it is considered a convenient and reproducible laboratory parameter. Therefore, if the clinical significance of posttreatment NLR is well defined, it can be a useful indicator of poor prognosis and may suggest further adjuvant treatment after definitive CCRT.

The main limitations of this study are its retrospective nature and the small sample size, which may have contributed to selection bias. In addition, it is a single-center study, and thus, the generalization of our findings is limited. Therefore, further large, prospective, and multicenter clinical studies are warranted to determine the significance of posttreatment NLR. Despite these limitations, our study offers 
A

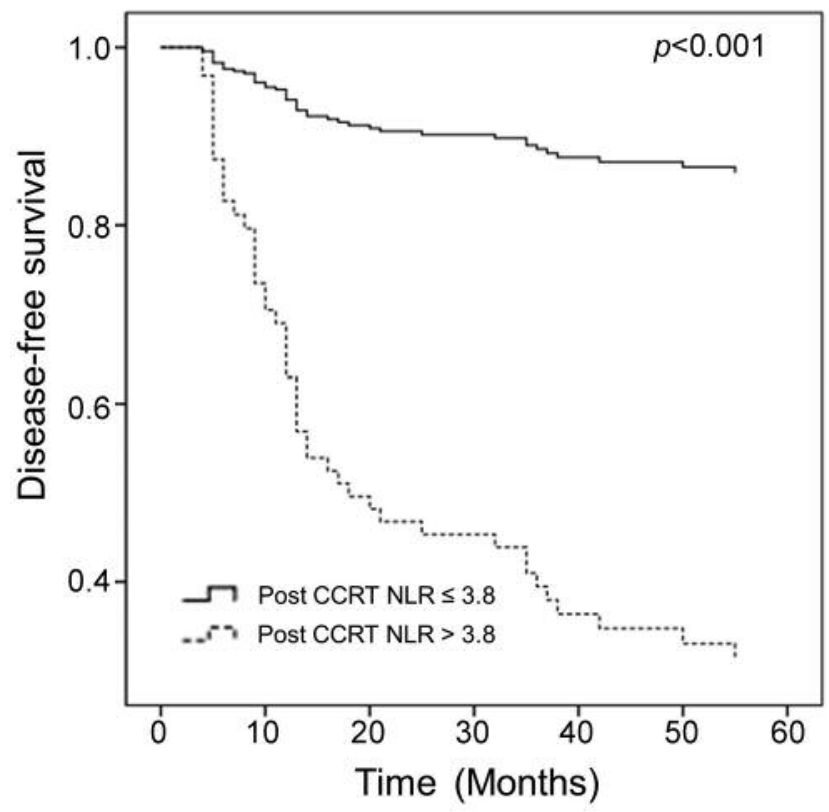

B

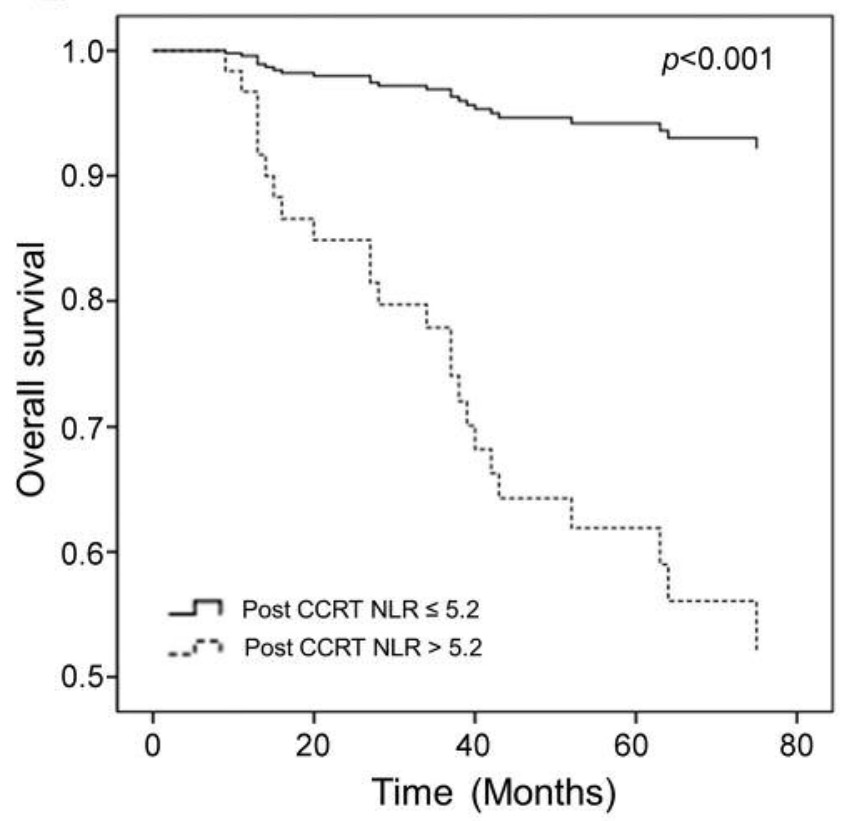

Figure 2. Survival curves for patients with high post-CCRT neutrophil-to-lymphocyte ratio (NLR). A. Disease-free survival, B. Overall survival.

some unique and significant findings. Unlike previous studies, no significant prognostic value of pre-treatment hematological parameters was observed. Furthermore, this is the first study to reveal the prognostic significance of posttreatment NLR in patients with LACC who were treated by definitive CCRT.

\section{Conclusion}

In summary, our study demonstrated that high post-treatment NLR is associated with poor DFS and OS in patients with LACC who received definitive CCRT. The post-treatment NLR is a convenient, simple, and reproducible hematological parameter, and therefore, it can be used for identifying patients at high risk of recurrence who may be benefit from further treatment and close surveillance.

\section{Conflicts of interest}

The Authors have no conflicts of interest to disclose in regard to this study.

\section{Authors' Contributions}

HJL and GOC designed the experiments, analyzed the data and prepared the manuscript. JMK, YJC, YHL, SHP, DGH and YSL performed data acquisition and made significant contributions to the drafting the manuscript. All Authors read and approved the final manuscript.

\section{References}

1 Bray F, Ferlay J, Soerjomataram I, Siegel RL, Torre LA and Jemal A: Global cancer statistics 2018: Globocan estimates of incidence and mortality worldwide for 36 cancers in 185 countries. CA Cancer J Clin 68(6): 394-424, 2018. PMID: 30207593. DOI: $10.3322 /$ caac. 21492

2 Endo D, Todo Y, Okamoto K, Minobe S, Kato H and Nishiyama $\mathrm{N}$ : Prognostic factors for patients with cervical cancer treated with concurrent chemoradiotherapy: A retrospective analysis in a japanese cohort. J Gynecol Oncol 26(1): 12-18, 2015. PMID: 25310853. DOI: $10.3802 /$ jgo.2015.26.1.12

3 Coussens LM and Werb Z: Inflammation and cancer. Nature 420(6917): 860-867, 2002. PMID: 12490959. DOI: 10.1038/ nature 01322

4 Smith RA, Bosonnet L, Raraty M, Sutton R, Neoptolemos JP, Campbell F and Ghaneh P: Preoperative platelet-lymphocyte ratio is an independent significant prognostic marker in resected pancreatic ductal adenocarcinoma. Am J Surg 197(4): 466-472, 2009. PMID: 18639229. DOI: 10.1016/j.amjsurg.2007.12.057

5 Leitch EF, Chakrabarti M, Crozier JE, McKee RF, Anderson JH, Horgan PG and McMillan DC: Comparison of the prognostic value of selected markers of the systemic inflammatory response in patients with colorectal cancer. Br J Cancer 97(9): 1266-1270, 2007. PMID: 17923866. DOI: 10.1038/sj.bjc.6604027

6 Teramukai S, Kitano T, Kishida Y, Kawahara M, Kubota K, Komuta K, Minato K, Mio T, Fujita Y, Yonei T, Nakano K, Tsuboi M, Shibata K, Furuse K and Fukushima M: Pretreatment neutrophil count as an independent prognostic factor in advanced non-small-cell lung cancer: An analysis of japan multinational trial organisation 1c00-03. Eur J Cancer 45(11): 1950-1958, 2009. PMID: 19231158. DOI: 10.1016/j.ejca.2009.01.023 
7 Wang L, Jia J, Lin L, Guo J, Ye X, Zheng X and Chen Y: Predictive value of hematological markers of systemic inflammation for managing cervical cancer. Oncotarget 8(27): 44824-44832, 2017. PMID: 28148894. DOI: 10.18632/oncotarget.14827

8 Onal C, Guler OC and Yildirim BA: Prognostic use of pretreatment hematologic parameters in patients receiving definitive chemoradiotherapy for cervical cancer. Int J Gynecol Cancer 26(6): 1169-1175, 2016. PMID: 27206286. DOI: 10.1097/IGC.0000000000000741

9 Nakamura K, Nishida T, Haruma T, Haraga J, Omichi C, Ogawa C, Kusumoto T, Seki N, Masuyama H and Hiramatsu Y: Pretreatment platelet-lymphocyte ratio is an independent predictor of cervical cancer recurrence following concurrent chemoradiation therapy. Mol Clin Oncol 3(5): 1001-1006, 2015. PMID: 26623040. DOI: 10.3892/mco.2015.595

10 Mizunuma M, Yokoyama Y, Futagami M, Aoki M, Takai Y and Mizunuma $\mathrm{H}$ : The pretreatment neutrophil-to-lymphocyte ratio predicts therapeutic response to radiation therapy and concurrent chemoradiation therapy in uterine cervical cancer. Int J Clin Oncol 20(5): 989-996, 2015. PMID: 25736530. DOI: 10.1007/s10147-015-0807-6

11 Lee YY, Choi CH, Kim HJ, Kim TJ, Lee JW, Lee JH, Bae DS and Kim BG: Pretreatment neutrophil:Lymphocyte ratio as a prognostic factor in cervical carcinoma. Anticancer Res 32(4): 1555-1561, 2012. PMID: 22493401.

12 Chen L, Zhang F, Sheng XG, Zhang SQ, Chen YT and Liu BW: Peripheral platelet/lymphocyte ratio predicts lymph node metastasis and acts as a superior prognostic factor for cervical cancer when combined with neutrophil: Lymphocyte. Medicine (Baltimore) 95(32): e4381, 2016. PMID: 27512849. DOI: 10.1097/MD.0000000000004381

13 Campian JL, Sarai G, Ye X, Marur S and Grossman SA: Association between severe treatment-related lymphopenia and progression-free survival in patients with newly diagnosed squamous cell head and neck cancer. Head Neck 36(12): 17471753, 2014. PMID: 24174270. DOI: 10.1002/hed.23535

14 Lin AJ, Gang M, Rao YJ, Campian J, Daly M, Gay H, Oppelt P, Jackson RS, Rich J, Paniello R, Zevallos J, Hallahan D, Adkins $\mathrm{D}$ and Thorstad W: Association of post-treatment lymphopenia and elevated neutrophil-to-lymphocyte ratio with poor clinical outcomes in patients with human papillomavirus-negative oropharyngeal cancers. JAMA Otolaryngol Head Neck Surg, 2019. PMID: 30920592. DOI: 10.1001/jamaoto.2019.0034

15 Contreras JA, Lin AJ, Weiner A, Speirs C, Samson P, Mullen D, Campian J, Bradley J, Roach M and Robinson C: Cardiac dose is associated with immunosuppression and poor survival in locally advanced non-small cell lung cancer. Radiother Oncol 128(3): 498504, 2018. PMID: 29859754. DOI: 10.1016/j.radonc.2018.05.017

16 Hyder J, Boggs DH, Hanna A, Suntharalingam M and Chuong MD: Changes in neutrophil-to-lymphocyte and platelet-tolymphocyte ratios during chemoradiation predict for survival and pathologic complete response in trimodality esophageal cancer patients. J Gastrointest Oncol 7(2): 189-195, 2016. PMID: 27034785. DOI: 10.3978/j.issn.2078-6891.2015.080
17 Kim DY, Kim IS, Park SG, Kim H, Choi YJ and Seol YM: Prognostic value of post-treatment neutrophil-lymphocyte ratio in head and neck squamous cell carcinoma treated by chemoradiotherapy. Auris Nasus Larynx 44(2): 199-204, 2017. PMID: 27269133. DOI: 10.1016/j.anl.2016.05.013

$18 \mathrm{Wu}$ J, Chen M, Liang C and Su W: Prognostic value of the pretreatment neutrophil-to-lymphocyte ratio in cervical cancer: A meta-analysis and systematic review. Oncotarget 8(8): 1340013412, 2017. PMID: 28077792. DOI: 10.18632/oncotarget.14541

19 Huang H, Liu Q, Zhu L, Zhang Y, Lu X, Wu Y and Liu L: Prognostic value of preoperative systemic immune-inflammation index in patients with cervical cancer. Sci Rep 9(1): 3284, 2019. PMID: 30824727. DOI: 10.1038/s41598-019-39150-0

20 Jeong MH, Kim H, Kim TH, Kim MH, Kim BJ and Ryu SY: Prognostic significance of pretreatment lymphocyte percentage and age at diagnosis in patients with locally advanced cervical cancer treated with definite radiotherapy. Obstet Gynecol Sci 62(1): 35-45, 2019. PMID: 30671392. DOI: 10.5468/ogs.2019.62.1.35

21 Pecorelli S: Revised figo staging for carcinoma of the vulva, cervix, and endometrium. Int J Gynaecol Obstet 105(2): 103104, 2009. PMID: 19367689. DOI: 10.1016/j.ijgo.2009.02.012

22 Mantovani A, Allavena P, Sica A and Balkwill F: Cancer-related inflammation. Nature 454(7203): 436-444, 2008. PMID: 18650914. DOI: $10.1038 /$ nature07205

23 Paramanathan A, Saxena A and Morris DL: A systematic review and meta-analysis on the impact of pre-operative neutrophil lymphocyte ratio on long term outcomes after curative intent resection of solid tumours. Surgical Oncology-Oxford 23(1): 3139, 2014. PMID: 24378193. DOI: 10.1016/j.suronc.2013.12.001

24 Huang QT, Man QQ, Hu J, Yang YL, Zhang YM, Wang W, Zhong $\mathrm{M}$ and $\mathrm{Yu} \mathrm{YH}$ : Prognostic significance of neutrophil-tolymphocyte ratio in cervical cancer: A systematic review and meta-analysis of observational studies. Oncotarget 8(10): 1675516764, 2017. PMID: 28187430. DOI: 10.18632/oncotarget.15157

25 Balkwill F and Mantovani A: Inflammation and cancer: Back to virchow? Lancet 357(9255): 539-545, 2001. PMID: 11229684. DOI: $10.1016 / \mathrm{S} 0140-6736(00) 04046-0$

26 el-Hag A and Clark RA: Immunosuppression by activated human neutrophils. Dependence on the myeloperoxidase system. J Immunol 139(7): 2406-2413, 1987. PMID: 2821114.

27 Martinez-Lostao L, Anel A and Pardo J: How do cytotoxic lymphocytes kill cancer cells? Clin Cancer Res 21(22): 5047-5056, 2015. PMID: 26567364. DOI: 10.1158/1078-0432.CCR-15-0685

28 Dunn GP, Old LJ and Schreiber RD: The immunobiology of cancer immunosurveillance and immunoediting. Immunity 21(2): 137-148, 2004. PMID: 15308095. DOI: 10.1016/j.immuni.2004.07.017

Received November 27, 2019

Revised December 3, 2019

Accepted December 4, 2019 